\title{
Liquid Chromatographic Determination of Alcohols in Food and Beverages with Indirect Polarimetric Detection Using a $\beta$-Cyclodextrin Mobile Phase
}

\author{
Edison Munaf,* Rahmiana ZeIn,* Ji-Ye Jin,** and Toyohide TAKeuCHI***† \\ *Department of Chemistry, Faculty of Mathematics and Natural Sciences, Andalas University, \\ Padang 25163, Indonesia \\ **Instrumental Analysis Center, Gifu University, 1-1 Yanagido, Gifu 501-1193, Japan \\ ***Department of Chemistry, Faculty of Engineering, Gifu University, 1-1 Yanagido, Gifu 501-1193, Japan
}

\begin{abstract}
An indirect polarimetric detection method for the determination of alcohols has been proposed in liquid chromatography (LC). Optically active mobile-phase additives, such as $\beta$-cyclodextrin $(\beta$-CD), could be used to visualize optically inactive alcohols in reversed-phase LC. The visualization of alcohols is based on a perturbation of the partition of $\beta$-CD caused by the alcohols. The detection limits of the present system at a signal-to-noise ratio of 3 were $0.031,0.019,0.018$, 0.013, 0.011, 0.008 and $0.008 \%$ (v/v) for ethanol, 2-propanol, 1-propanol, 2-methyl-2-propanol, 2-butanol, 2-methyl-1propanol and 1-butanol, respectively. The method was successfully applied to the determination of ethanol present in food and beverage samples.
\end{abstract}

(Received February 14, 2002; Accepted July 4, 2002)

\section{Introduction}

The determination of alcohols is of great importance in clinical and environmental analysis as well as in the food and beverage industries. A number of methods have been developed to determine the alcohol concentration in various samples. Methods such as enzymatic ${ }^{1}$ and non-enzymatic gas diffusion, ${ }^{2}$ amperometric flow-injection analysis, ${ }^{3}$ colorimetry using a dehydrogenase-based biosensor ${ }^{4}$ and gas chromatography (GC), ${ }^{5,6}$ have been well described. Among these methods, a direct GC method coupled with a flame ionization detector and a capillary column is the most widely used techniques for the determination of alcohols in various matrices.

To improve the detection sensitivity and the selectivity, fluorescence agents, such as $p$ - $N, N$-dialkylaminotrifluoroacetylstilbenes, ${ }^{7}$ 4-nitrobenzoyl chloride, ${ }^{8}$ acridone-9- $N$ acetylbenzenedisulfonate, ${ }^{9} \quad$ 5-(4-aminophneyl)-2-(2-pyrazinyl)1,3 -oxazole, ${ }^{10}$ have been applied to the quantification of alcohols in fluorometry as well as in liquid chromatography (LC) with precolumn or postcolumn derivatization techniques. Although these methods are sensitive, they involve rather complicated processes, especially in the derivatization.

Indirect detection systems have been described in LC for the speciation and determination of chemical species. ${ }^{11-16}$ In general, when the analyte transfers the visualization reagent molecules from the stationary phase to the mobile phase and vice versa, the analyte could be indirectly detected due to a change in the background signal of the eluent. Nonionic compounds, such as alcohols, can be indirectly detected in reversed-phase LC, where analytes are visualized by their

$\dagger$ To whom correspondence should be addressed.

E-mail: takeuchi@apchem.gifu-u.ac.jp perturbation of the partition of a mobile-phase additive. ${ }^{12} \mathrm{~A}$ previous paper proved the detection mechanism involved in the indirect detection of nonionic compounds based on the perturbation of partitioning. ${ }^{12}$ The larger is the dynamic reserve, the better is the sensitivity that can be achieved.

Optically active detection, such as polarimetry, is one of the most prominent indirect detection methods for LC because it can maintain a higher dynamic reserve, defined as the ratio of the background to its noise level. ${ }^{13}$ Bobbitt and Yeung ${ }^{13}$ presented results on indirect polarimetric detection in microbore LC using a laboratory-made flow cell and an argon-ion laser as a light source. They achieved a mass detection limit of $390 \mathrm{ng}$ at $S / N=3$ for an optically inactive solute, such as dodecane, corresponding to a concentration detection limit of $0.078 \%$.

To our knowledge, the present paper describes indirect polarimetric detection for the first time in the literature using a commercially available optically active detector. The feasibility of indirect polarimetric detection of alcohols and its application to the determination of alcohols in foods and beverages will be shown.

\section{Experimental}

Reagents

The reagents used in this work were of analytical reagent grade, and were obtained from Nacalai Tesque (Kyoto, Japan), unless otherwise noted. $\alpha$-Cyclodextrin $(\alpha-\mathrm{CD}), \beta-\mathrm{CD}, \gamma-\mathrm{CD}$, methanol, ethanol, 2-propanol, 1-propanol, 2-methyl-2-propanol, 2-butanol, 2-methyl-1-propanol and 1-butanol were used as received. Purified water was prepared in the laboratory using a GS-590 water distillation system (ADVANTEC, Tokyo, Japan).

The eluents were degassed under a vacuum using an ultrasonic vibration bath before use. 


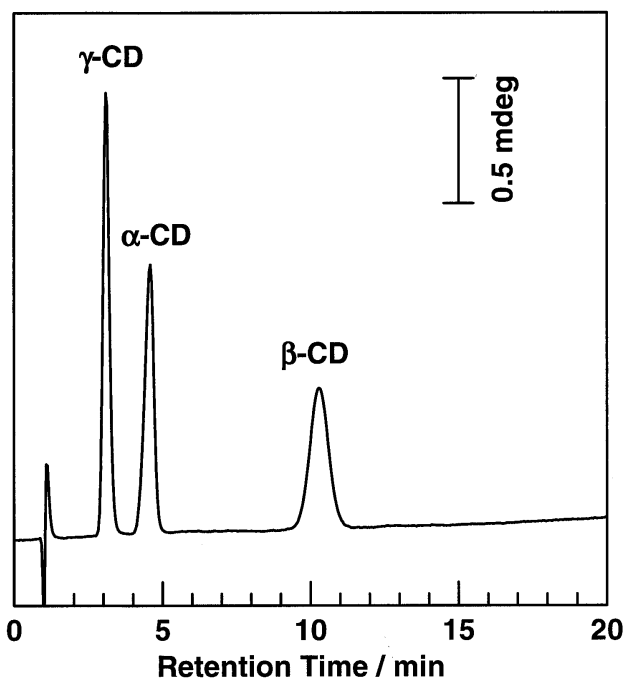

Fig. 1 Detection of $\alpha-, \beta$ - and $\gamma$-CD using $5 \%$ methanol as the mobile phase. Column, Develosil ODS-UG-5 $(50 \times 4.6 \mathrm{~mm}$ i.d.); eluent, $5 \%$ methanol aqueous solution; flow rate, $1 \mathrm{~mL} \mathrm{~min}{ }^{-1}$; injection volume, $21 \mu \mathrm{L}$; analyte, $0.1 \%$ (w/v) each for $\alpha$-CD and $\gamma$ $\mathrm{CD}$; detector, optical rotatory detector.

\section{Apparatus}

The chromatographic system consisted of a Model 880-PU intelligent HPLC pump, (Jasco, Tokyo, Japan), a Model 7700 injection valve (Rheodyne, Cotati, CA, USA) coupled with a 21 $\mu \mathrm{L}$ loop, a 50 or $150 \times 4.6 \mathrm{~mm}$ i.d. Develosil ODS-UG-5 packed column ( $5 \mu \mathrm{m}$; Nomura Chemical, Seto, Japan), a Model OR-990 optically rotatory dispersion detector (ORD; Jasco), and a Chromatopac C-R7Ae plus data processor (Shimadzu, Kyoto, Japan). The separation column was immersed in a water bath to avoid variation in the ambient temperature. The flow rate of the mobile phase was kept constant at $1.0 \mathrm{~mL} / \mathrm{min}$. The temperature of the water-bath was not regulated, but left ambient $\left(\mathrm{ca} .20^{\circ} \mathrm{C}\right)$.

\section{Preparation of sample}

A beer sample was filled in a $250 \mathrm{~mL}$ glass bottle and poured 3 times from one bottle into another to decrease the amount of foam. Afterwards, it was degassed under a vacuum and diluted 10 times with purified water prior to injection. Wine samples were directly injected after 10 -fold dilution with purified water. Traditional West Sumatra Indonesian foods, called tapai ubi (fermented from cassava) and tapai pulut (fermented from sticky rice), were pressed to remove any liquid, and then filtered through a $0.45-\mu \mathrm{m}$ membrane filter (GL Sciences, Tokyo, Japan). The filtrates were then diluted 10 times with purified water prior to the injection.

\section{Results and Discussion}

\section{Selection of an optically active mobile-phase additive}

The mobile-phase additive should be selected while considering its retentivity and response ability to the detector. The analyte signal intensity in indirect detection depends on both the retention factor of the mobile-phase additive $\left(k_{\mathrm{ad}}\right)$ and that of the analyte $\left(k_{\mathrm{an}}\right)$, as shown by the following equation: ${ }^{12}$

$$
\text { Peak height } \propto k_{\text {ad }} /\left|k_{\text {an }}-k_{\text {ad }}\right| \text {. }
$$

Equation (1) indicates that the analyte eluting close to the

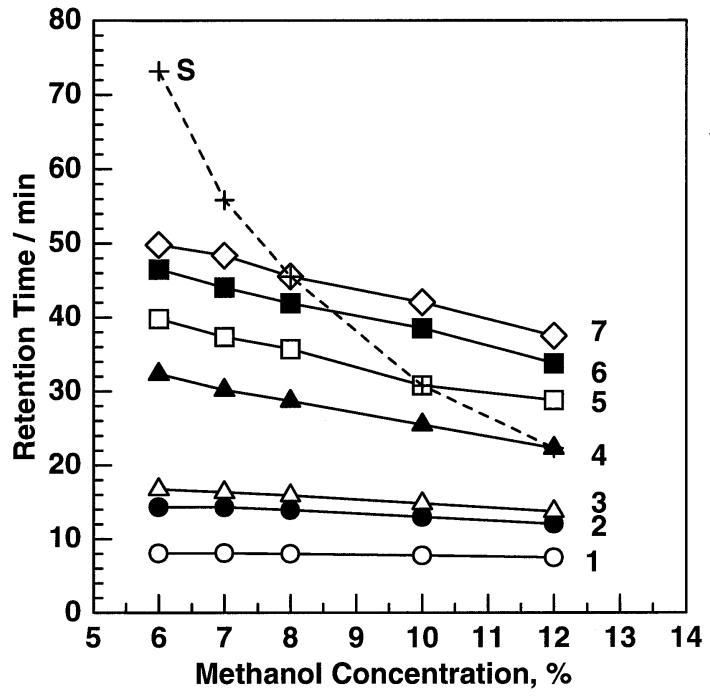

Fig. 2 Effect of the methanol concentration in the mobile phase on the retention time of alcohols. Column, Develosil ODS-UG-5 $(150 \times$ $4.6 \mathrm{~mm}$ i.d.); eluent, methanol containing $0.1 \%(\mathrm{w} / \mathrm{v}) \quad \beta-\mathrm{CD}$; analytes, 1 = ethanol, 2 = 2-propanol, 3 = 1-propanol, 4 = 2-methyl2-propanol; 5 = 2-butanol, $6=2$-methyl-1-propanol, $7=1$-butanol, $\mathrm{S}$ $=$ system peak; other operating conditions as in Fig. 1 .

system peak has a potential to give a large signal. It is also expected that the peak height of the analyte increases with increasing $k_{\text {ad }}$. The system peak appears at $k_{\text {ad }}$, and the peak direction, positive or negative, depends on the relative retention of the analyte to the system peak. It is shown that when the analyte decreases the retention factor of the mobile phase additive, the analyte eluting before the system peak gives a positive peak, whereas the analyte eluting after the system peak gives a negative peak. ${ }^{12}$ On the contrary, when the analyte increases the retention factor of the additive, the analyte eluting before the system peak gives a negative peak, whereas the analyte eluting after the system peak gives a positive peak.

Alcohols can be separated in the reversed-phase mode using eluents containing a low concentration of organic solvents, such as acetonitrile or methanol. CDs are candidates for the mobilephase additive because the retentivity in the reversed-phase mode is nearly the same as that of the alcohols. Figure 1 demonstrates the separation of native CDs using a $5 \%(\mathrm{v} / \mathrm{v})$ methanol aqueous solution as the mobile phase. It can be seen that $\alpha$-, $\beta$ - and $\gamma$-CD can be separated well within 10 min. As can be expected from Eq. (1), the analyte signal increases when the retention of the mobile phase additive increases and/or the additive elutes close to analytes. Therefore, the analyte signal strongly depends on the retention of the additive. Considering the result in Fig. 1 and Eq. (1), $\beta$-CD is selected as the optically active mobile-phase additive for the indirect polarimetric detection of alcohols in LC because it gives the best sensitivity among the examined CDs. In addition, the specific rotation of $\mathrm{CD}$ is +160 degrees.

\section{Effect of the methanol concentration in the mobile phases}

Alcohols could be visualized by using aqueous methanol solution containing $\beta$-CD as the mobile-phase additive. The effects of the methanol concentration in the mobile phases on the retention of ethanol, 1-propanol, 2-propanol, 2-methyl-2propanol, 1-butanol, 2-butanol and 2-methyl-1-propanol were examined in the presence of $0.1 \%(\mathrm{w} / \mathrm{v}) \beta$-CD. The results are shown in Fig. 2. The results indicate that the retention of 


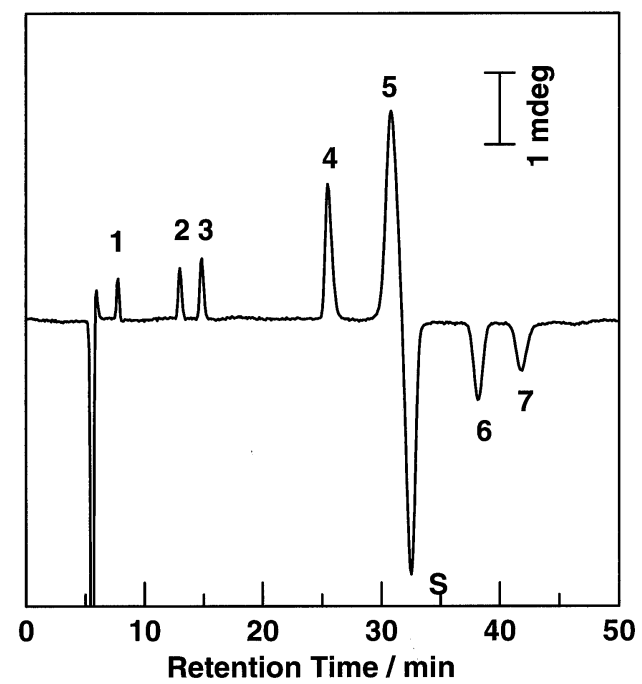

Fig. 3 Separation of alcohols using a 10\% (v/v) methanol aqueous solution containing $0.1 \%(\mathrm{w} / \mathrm{v}) \quad \beta$-CD as the mobile phase. Eluent, $10 \%(\mathrm{v} / \mathrm{v})$ methanol aqueous solution containing $0.1 \%(\mathrm{w} / \mathrm{v}) \quad \beta-\mathrm{CD}$ analytes $(\%, \mathrm{v} / \mathrm{v}), 1=$ ethanol $(0.2), 2=2$-propanol $(0.1), 3=1$ propanol (0.1), $4=2$-methyl-2-propanol (0.05), $5=2$-butanol (0.05), 6 = 2-methyl-1-propanol (0.05), 7 = 1-butanol (0.05); other operating conditions as in Fig. 2.

alcohols decreases with increasing methanol concentration in the mobile phase. It should be noted that the retention of the system peak of $\beta$-CD also decreases with increasing methanol concentration. When the methanol concentration was higher than $8 \%(\mathrm{v} / \mathrm{v})$, the system peak due to $\beta$-CD overlapped with the analytes.

Figure 3 demonstrates the indirect detection of alcohols using a $10 \%(\mathrm{v} / \mathrm{v})$ methanol aqueous solution containing $0.1 \%(\mathrm{w} / \mathrm{v})$ $\beta$-CD as the mobile phase. It is seen that the system peak overlaps with 2-butanol, and the peak direction changes between before and after the system peak, as can be expected from the above discussion. As shown in Fig. 3, positive peaks are observed for the analytes eluting before the system peak, whereas negative peaks are observed for the analyte eluting after the system peak. These results mean that the analytes decrease the retentivity of $\beta$-CD. The system peak should be eluted so as not to interfere with the analyte peaks of interest.

Under the conditions in Fig. 3, the background signal of the mobile phase is $+1.3 \times 10^{-2}$ degrees, whereas the noise level is $1.5 \times 10^{-5} \mathrm{deg}$. The dynamic reserve is then calculated to be 880 , which is lower than expected. The dynamic reserves for common detection, such as UV and fluorimetry, are around $10^{4}$ and $10^{3}$, respectively. ${ }^{14}$ The dynamic reserve of the present system is about 10-times lower than that of a UV detection system, which means that the sensitivity of the present system is about 10-times worse than that of indirect UV detection.

If the dynamic reserve can be increased, the sensitivity of the present detection method can be improved. The dynamic reserve of the present detection system will be improved by increasing the concentration of optically active additive agents, or selecting a reagent with a higher specific optical rotation because the OR-990 detector can measure up to 0.5 degrees. The dynamic reserve is generally improved by increasing the background signal while maintaining the noise level.

\section{Effects of the $\beta$-CD concentration in the mobile phases}

The effect of the $\beta$-CD concentration in the mobile phase on

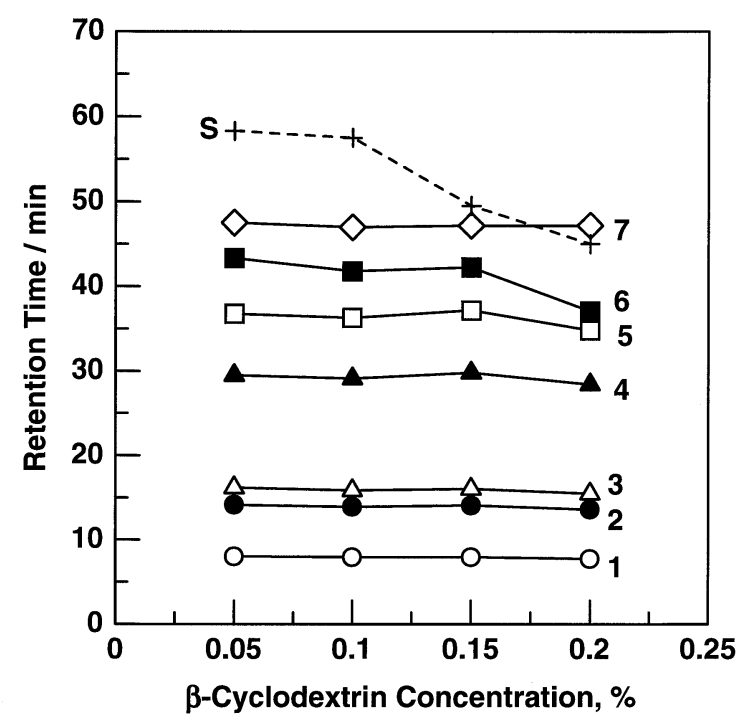

Fig. 4 Effect of the $\beta$-CD concentration in the mobile phase on the retention time of the alcohols. Eluent, $\beta-\mathrm{CD}$ in $7 \%(\mathrm{v} / \mathrm{v})$ methanol; other operating conditions as in Fig. 2.

the retention of alcohols was examined. The results are shown in Fig. 4, where the concentration of methanol in the mobile phase is kept constant at $7 \%(\mathrm{v} / \mathrm{v})$. It was observed that when the concentration of $\beta$-CD was changed from 0.05 to $0.2 \%$ $(w / v)$, the retention of alcohols was almost constant. On the other hand, the signal intensity of the analyte peak increased with increasing the $\beta$-CD concentration. However, the noise also increased with increasing the $\beta$-CD concentration, e.g., the noise for $0.2 \%(\mathrm{w} / \mathrm{v}) \beta$-CD was about twice that for the case of $0.1 \% \beta$-CD. This means that the dynamic reserve cannot be improved by increasing the $\beta$-CD concentration to higher than $0.1 \%(\mathrm{w} / \mathrm{v})$. From these results, $0.1 \%(\mathrm{w} / \mathrm{v})$ was selected as the concentration of $\beta$-CD in the mobile phase.

\section{Linear dynamic range}

Figure 5 demonstrates the separation of ethanol, 1-propanol, 2-propanol, 2-methyl-2-propanol, 1-butanol, 2-butanol and 2methyl-1-propanol on the ODS column using a $7 \%(\mathrm{v} / \mathrm{v})$ methanol aqueous solution containing $0.1 \%(\mathrm{w} / \mathrm{v}) \beta-\mathrm{CD}$ as the mobile phase. Although it took 90 min to elute the system peak, the analytes were completely separated.

The linearity of the signal was examined under the conditions given in Fig. 5; the results are given in Table 1. The present methods can provide quantitative information about alcohols, although the linear range is not very wide. UV and fluorescence detectors normally achieve two to three orders of the dynamic range. The detection limits of the analytes at $S / N=3$ are also listed in Table 1: 0.031, 0.019, 0.018, 0.013, 0.011, 0.008 and $0.008 \%(\mathrm{v} / \mathrm{v})$ for ethanol, 2-propanol, 1-propanol, 2-methyl-2propanol, 2-butanol, 2-methyl-1-propanol and 1-butanol, respectively. The detection limit of an optically inactive analyte achieved by Bobbitt and Yeung ${ }^{13}$ was $0.078 \%$. It should be noted again that the present system can improve the detection limits by selecting a visualization agent and/or by adjusting the mobile-phase conditions.

\section{Application to the analysis of alcohol in foods and beverages}

The present system was applied to the determination of alcohols contained in Indonesian traditional foods, e.g., tapai ubi (fermented from cassava) and tapai pulut (fermented from 


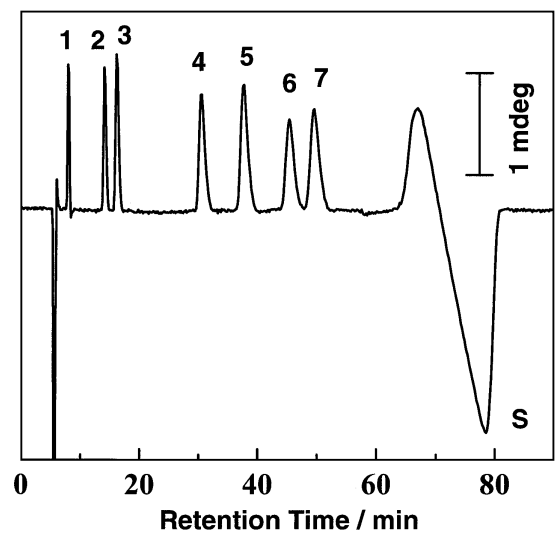

Fig. 5 Detection of seven alcohols. Operating conditions as in Fig. 2 , except for the analyte concentration. Analyte $(\%, \mathrm{v} / \mathrm{v}): 1=$ ethanol (0.6), 2 = 2-propanol (0.3); 3 = 1-propanol (0.3), 4 = 2-methyl-2propanol $(0.15) ; 5=2$-butanol $(0.15), 6=2$-methyl-1-propanol (0.075), 7 = 1-butanol (0.075).

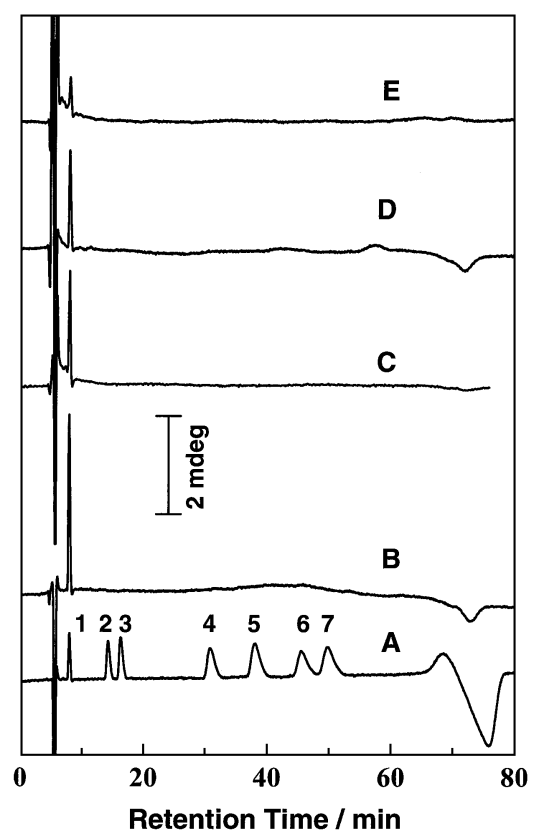

Fig. 6 Detection of ethanol in Indonesian traditional foods and beverages using an optical rotatory detector. Eluent, $7 \%(\mathrm{v} / \mathrm{v})$ methanol containing $0.1 \%(\mathrm{w} / \mathrm{v}) \quad \beta$-CD; other operating conditions as in Fig. 2 except for the sample. Samples: A, authentic mixture of alcohols $(\%, \mathrm{v} / \mathrm{v}), 1=$ ethanol $(0.4), 2=2$-propanol $(0.2), 3=1$ propanol (0.2), $4=2$-methyl-2-propanol (0.1), $5=2$-butanol $(0.1), 6$ = 2-methyl-1-propanol (0.05), 7 = 1-butanol $(0.05)$; B, wine; $\mathrm{C}$, beer; $\mathrm{D}$, tapai ubi; E, tapai pulut.

sticky rice) as well as to wine and beer. All of the samples were diluted 10 times prior to injection. The chromatograms are demonstrated in Fig. 6. From the figure it can be seen that ethanol present in foods and beverages can be detected. The ethanol concentrations were 16, 6.3, 6.3 and $2.8 \%(\mathrm{v} / \mathrm{v})$ for wine, beer, tapai ubi and tapai pulut, respectively. Other alcohols were lower than the detection limits of the present system. Although it is known that alcohols other than ethanol are contained in alcoholic beverages, their concentrations are lower than the detection limits achieved by the present detection system.
Table 1 Linear dynamic range of alcohols for indirect polarimetric detection using a Develosil ODS-UG-5 $(150 \times 4.6$ $\mathrm{mm}$ i.d.) separation column and $7 \%(\mathrm{v} / \mathrm{v})$ methanol containing $0.1 \%(\mathrm{w} / \mathrm{v}) \beta$-CD as the mobile phase

\begin{tabular}{|c|c|c|c|c|c|}
\hline \multirow{2}{*}{ Analyte } & \multirow{2}{*}{$\begin{array}{c}\text { Linearity range } \\
(\%, \mathrm{w} / \mathrm{v})\end{array}$} & \multicolumn{3}{|c|}{$\mathrm{RSD}, \%^{\mathrm{a}}$} & \multirow{2}{*}{$\begin{array}{l}\mathrm{LOD}^{\mathrm{b}} \\
(\%, \mathrm{v} / \mathrm{v})\end{array}$} \\
\hline & & $\mathrm{RT}^{\mathrm{b}}$ & $\mathrm{RH}^{\mathrm{b}}$ & $\mathrm{RA}^{\mathrm{b}}$ & \\
\hline Ethanol & $0.2-0.8$ & 1.8 & 1.7 & 1.9 & 0.031 \\
\hline 2-Propanol & 0.1 & 1.9 & 2.1 & 2.3 & 0.019 \\
\hline 1-Propanol & $0.1-0.4$ & 2.1 & 2.1 & 2.8 & 0.018 \\
\hline 2-Methyl-2-propanol & $0.05-0.2$ & 2.3 & 2.3 & 3.4 & 0.013 \\
\hline 2-Butanol & $0.05-0.2$ & 2.2 & 3.2 & 2.9 & 0.011 \\
\hline 2-Methyl-2-propanol & $0.025-0.1$ & 1.9 & 3.4 & 3.7 & 0.008 \\
\hline 1-Butanol & $0.025-0.1$ & 2.4 & 4.1 & 4.3 & 0.008 \\
\hline
\end{tabular}

a. Based on three successive measurements.

b. RSD, relative standard deviation; RT, retention time; PH, peak height; PA, peak area; LOD, limit of detection.

In conclusion, the method could be applicable to the determination of ethanol contained in foods and beverages. The sensitivity of the present detection system will be improved by selecting a reagent with a higher specific rotation and by reducing the noise level. The present detection system is universal rather than selective.

\section{Acknowledgements}

One of the author (E. M.) would like to express his grateful acknowledgement to the Japan Society for the Promotion of Sciences (JSPS), for the fellowship offer during the course of this work (ID No. S-01242).

\section{References}

1. Y. Sekine, M. Suzuki, T. Takeuchi, E. Tamiya, and I. Karube, Anal. Chim. Acta, 1993, 280, 179.

2. H. Ohura, T. Imato, Y. Asano, S. Yamasaki, and N. Ishibashi, Anal. Sci., 1990, 6, 541.

3. W. Kunnecke and R. D. Schmid, Anal. Chim. Acta, 1990, 234, 213.

4. T. Yao, Y. Kobayashi, and S. Musha, Anal. Chim. Acta, 1982, 139, 363.

5. S.-T. Cheung and W.-N. Lin, J. Chromatogr., 1987, 414, 248.

6. H. T. Nguyen, N. Takenaka, H. Bandow, and Y. Maeda, Anal. Sci., 2001, 17, 639.

7. G. J. Mohr, F. Lehmann, U.-W. Grummt, and U. E. Spichiger-Keller, Anal. Chim. Acta, 1997, 344, 215.

8. R. Schwarzenbach, J. Chromatogr., 1977, 140, 304.

9. J. You, X. Sun, W. Lao, Q. Ou, and D. Jiang, Anal. Chim. Acta, 1999, 391, 43.

10. G. Orellana, A. M. Gómez-Carneros, C. de Dios, A. A. García Martínez, and M. C. Moreno-Bondi, Anal. Chem., 1995, 67, 2231.

11. H. Small and T. E. Miller, Jr., Anal. Chem., 1982, 54, 462.

12. T. Takeuchi, S. Watanabe, K. Murase, and D. Ishii, Chromatographia, 1988, 25, 107.

13. D. R. Bobbitt and E. S. Yeung, Anal. Chem., 1984, 56, 1577.

14. T. Takeuchi and E. S. Yeung, J. Chromatogr., 1986, 366, 145 .

15. G. Schill and J. Crommen, Tr. Anal. Chem., 1987, 6, 111.

16. T. Takeuchi, Bunseki Kagaku, 2000, 49, 649. 